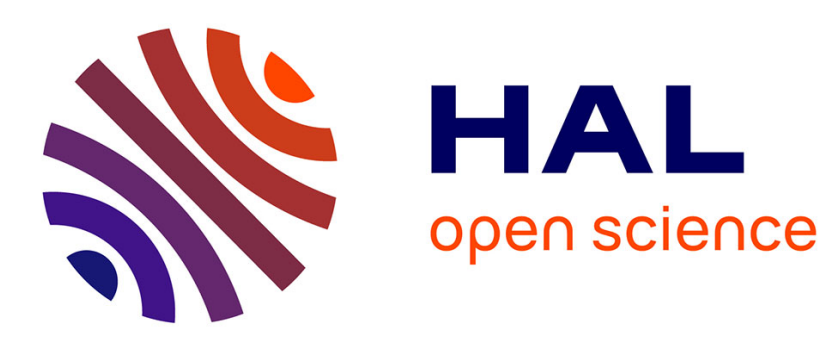

\title{
Sensitivity Analysis of Microstrip Line Parameters on Total Radiated Power Through Surrogate Modelling
}

Florent Delaporte, Philippe Besnier, Béatrice Azanowsky

\section{To cite this version:}

Florent Delaporte, Philippe Besnier, Béatrice Azanowsky. Sensitivity Analysis of Microstrip Line Parameters on Total Radiated Power Through Surrogate Modelling. 2019 International Symposium on Electromagnetic Compatibility, EMC EUROPE, Sep 2019, Barcelone, Spain. hal-02314002

\author{
HAL Id: hal-02314002 \\ https://hal.science/hal-02314002
}

Submitted on 11 Oct 2019

HAL is a multi-disciplinary open access archive for the deposit and dissemination of scientific research documents, whether they are published or not. The documents may come from teaching and research institutions in France or abroad, or from public or private research centers.
L'archive ouverte pluridisciplinaire HAL, est destinée au dépôt et à la diffusion de documents scientifiques de niveau recherche, publiés ou non, émanant des établissements d'enseignement et de recherche français ou étrangers, des laboratoires publics ou privés. 


\title{
Sensitivity Analysis of Microstrip Line Parameters on Total Radiated Power Through Surrogate Modelling
}

\author{
Florent Delaporte ${ }^{1,2}$, Philippe Besnier ${ }^{1}$, Béatrice Azanowsky ${ }^{2}$ \\ ${ }^{1}$ INSA Rennes, CNRS, IETR - UMR 6164, F-35000, Rennes, France \\ ${ }^{2}$ Thales, Cholet, France
}

\begin{abstract}
EMC standards are defined at system level. A system is composed of equipment that include printed circuit boards. Lowering the total radiated power by the latter contributes to minimize the risk to overcome the radiated emissivity limits at system level. We aim at optimizing printed circuit board design with regard to total radiated power while fulfilling other constraints such as signal integrity. Impact of electrical tracks topology on radiated field should be controlled. Electromagnetic solvers help to do so, but deal with some shortcomings. First, the output of a model of radiated power from a conductive track must be close enough from the actual radiated power of the corresponding printed track. Second, a parametric study is out of reach since we face a large number of variables. This paper presents a methodology to overcome these obstacles. We first assume that the model response and measurements are sufficiently correlated. We check this assumption for a few test cases. Then, the radiated power of a microstrip line is evaluated according to its parameters and frequency through a surrogate model built from a limited set of simulations. At the end, critical parameters are identified. We apply this methodology to a simple scenario for illustration.
\end{abstract}

Keywords-Emissivity, Total Radiated Power, Printed Circuit Board, Surrogate model, Sensitivity Analysis

\section{INTRODUCTION}

We deal in this paper with the problem of limiting the total radiated power of a printed circuit board (PCB). Since we have no control of the radiated emission of the system in which the PCB is integrated, we focus on minimizing the intrinsic total radiated power (RP) of the stand-alone PCB. The simple reasoning behind is that it also contributes to the limitation of the radiated emissivity of the system according to the relevant standard. However, a complex PCB contains hundreds of tracks, requiring too much calculation, preprocessing and post-processing to analyze an entire PCB. Risky tracks have to be identified in a pre-study. A list of design parameters and typical scenarii was defined to determine which are critical and must be analyzed. This paper presents one of these scenarii, the simplest one, which consists of a straight microstrip line parametrized by its length $(L)$, its width (w) and its distance to ground plane $(h)$. The frequency $(f)$ and load capacity $(C)$ at the far-end of the line (the near-end is the $50 \Omega$ impedance of the vector network analyzer) are also taken into account. The objective of this case study is to determine the RP as a function of the parameters previously cited:

$$
R P=\phi(L, w, h, C, f)
$$

The first part of this paper presents in detail the case study. To start this investigation a model of radiation is needed. Too many realizations are necessary to make measurements. Therefore, we make use of an electromagnetic solver. In order to check that the model simulated is accurate enough, some simulated configurations are compared to measurements. The methodology to carry out the comparison is detailed in section III: the choice of the solver and some of the tuning of its endogenous parameters are discussed, the technique employed to make measurements is detailed and the results are then shown. After validation of the simulation model, it can be exploited to carry out a parametric study. However, the number of simulations needed is still too large. Resorting to a surrogate model is inevitable. The chosen kriging surrogate model is presented in section IV and its self-consistency is verified with some extra simulations. Then, it can be safely exploited to make a sensitivity analysis, which is performed in section V.

\section{CASE STUDY}

The case studied here contains the most fundamental parameters of a simple microstrip line. It has 5 parameters (see Fig. 1): line length $(L)$, line width $(w)$, dielectric height $(h)$, load capacitance $(C)$ and frequency $(f)$. Frequency and load capacitance are input constraints of the design and not adjustable parameters but their influence must be assessed to determine the tracks with a potential risk on the PCB, and then focus on it. The definition interval of $\phi$ in (1) is described in TABLE I.

TABLE I. VALUES' INTERVAL FOR PARAMETERS

\begin{tabular}{|l|l|l|}
\hline \multicolumn{1}{|c|}{ Parameters } & \multicolumn{1}{|c|}{ Minimum value } & Maximum value \\
\hline $\mathrm{L}$ & $2 \mathrm{~cm}$ & $20 \mathrm{~cm}$ \\
\hline $\mathrm{w}$ & $100 \mu \mathrm{m}$ & $600 \mu \mathrm{m}$ \\
\hline $\mathrm{h}$ & $80 \mu \mathrm{m}$ & $800 \mu \mathrm{m}$ \\
\hline $\mathrm{C}$ & $1 \mathrm{pF}$ & $20 \mathrm{pF}$ \\
\hline $\mathrm{f}$ & $200 \mathrm{MHz}$ & $2 \mathrm{GHz}$ \\
\hline
\end{tabular}

The microstrip line is fed by a SMA connector and loaded by another SMA connector, which is plugged into another small PCB where a capacitor is mounted. In the simulation, the capacitance was considered as a perfect 
capacitance to limit the number of parameter to study. However, in part III.C a RLC series model is used for a better representation of the actual capacitor. It is extracted from measurement with the 2-port shunt thru method presented in [1].

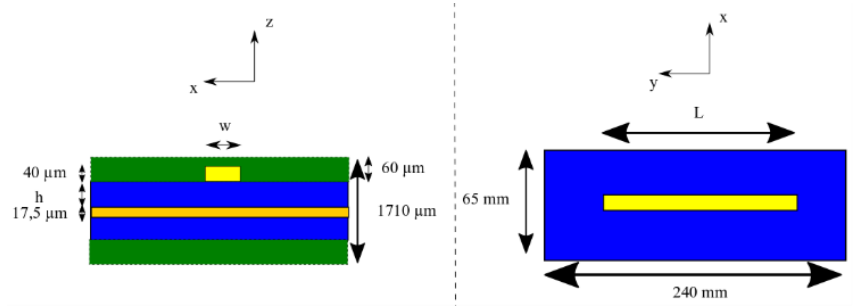

Fig. 1. Dimensions of the case study

\section{Simulation Vs MeAsuremENT}

\section{A. Full-Wave simulation}

We have carried out this case study using the finite integration technique (FIT) solver of CST MICROWAVE STUDIO ${ }^{\circledR}$ software. We chose this software since it is a time domain solver, allowing broadband simulation. Such a full wave solver also provides better accuracy, particularly in this case where the termination is not small with regard to the wavelength and cannot be modeled in $2.5 \mathrm{D}$ solvers. However full wave solvers are time consuming, so caution was taken to have a sufficient meshing (at least around 4 million meshes) and time windows limited to $21 \mathrm{~ns}$ to reach satisfying results in the minimum of time. Forty minutes are necessary to simulate a single $L, w, h, C$ configuration, which justify the use of a surrogate model to evaluate the function (1). The radiated field is obtained by Fourier transform of the field coming out of the simulation volume surrounded by a perfect matched layer. Then the result is integrated on the surface to obtain the RP (post-processing automatically made by the software). The result is normalized by the software to 0.5 watt for all frequency point, to be independent of the spectrum of the excitation signal.

To ensure a fair comparison with the measurement of the fabricated PCBs, the design (made on Cadence ${ }^{\circledR}$ ) was exported in CST MICROWAVE STUDIO $\AA$ and modified to be parametric. Radiated power is collected from simulation with several sets of random values of all parameters to train the surrogate model.

\section{B. Measurements}

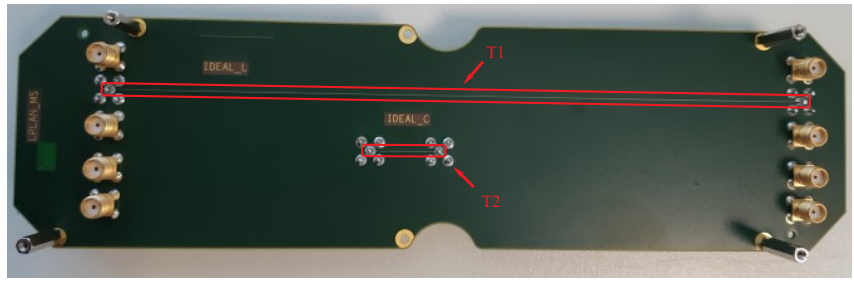

Fig. 2. Picture of the board with scenario $\mathrm{T} 1$ and $\mathrm{T} 2$

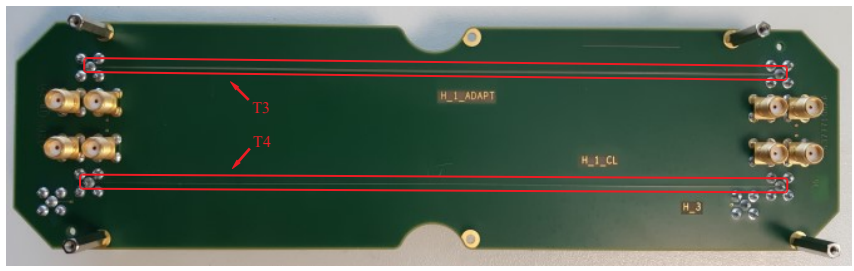

Fig. 3. Picture of the board with scenario T3 and T4
To validate the simulation model it is necessary to check with some cases if the simulation gives consistent results with regard to measurements. To do so, some RP measurements are performed in Reverberation Chamber (RC), which is an appropriate environment to sense the power density generated from a source of EM field. The method, inspired from [2], uses a vector network analyzer to measure power transmitted from the DUT to a log-periodic antenna. The efficiency of the antenna is supposed to be constant in the frequency band and equal to 0.9. A second identical antenna is first used to evaluate the quality factor of the chamber. This factor is necessary to evaluate the RP from the transmission coefficient. Due to the size of the chamber and the functional band of the log-periodic antennas, the study is carried out in the $200 \mathrm{MHz}-2 \mathrm{GHz}$ frequency range with 9001 points. The bandwidth of the filter is set to $1 \mathrm{kHz}$ and the power injected by the network analyzer is constant $(0$ $\mathrm{dBm})$. Fifty positions of stirrer are used to perform an ensemble average of the field. A cable of $11 \mathrm{~cm}$ with ferrites integrated feeds the board to limit its radiation, as it is not represented in the simulation. The RP on 4 tracks distributed on 2 boards (Fig. 2 and Fig. 3) is measured for 2 load configurations at the far end: a capacitance (as described in case study) and a matched load. TABLE II. presents the $L, w$, and $h$ values of each scenario. Scenario T1 may be seen as a reference transmission line with nominal values of parameters $L, w$, and $h$ regarding other scenarii. Scenario T2 shows the effect of the length. Scenario T3 allows the study of the effect of dielectric height. Scenario T4 gives information about the mismatching of the line (all the other tracks are $50 \Omega$ ). Its characteristic impedance is about $94 \Omega$. The values of the RLC parameters of capacitive load, measured with the method presented in [1], are $\mathrm{R}=232 \mathrm{~m} \Omega$, $\mathrm{L}=562 \mathrm{pH}$ and $\mathrm{C}=10 \mathrm{pF}$.

TABLE II. VALUES OF PARAMETERS FOR EACH SCENARIO

\begin{tabular}{|c|c|c|c|}
\hline \multirow{2}{*}{ Scenario } & \multicolumn{3}{|c|}{ Parameters } \\
\cline { 2 - 4 } & $\boldsymbol{L}(\mathbf{m m})$ & $\boldsymbol{h}(\boldsymbol{\mu m})$ & $\boldsymbol{w}(\boldsymbol{\mu m})$ \\
\hline T1 & 200 & 100 & 150 \\
\hline T2 & 20 & 100 & 150 \\
\hline T3 & 200 & 300 & 590 \\
\hline T4 & 200 & 300 & 120 \\
\hline
\end{tabular}

C. Results

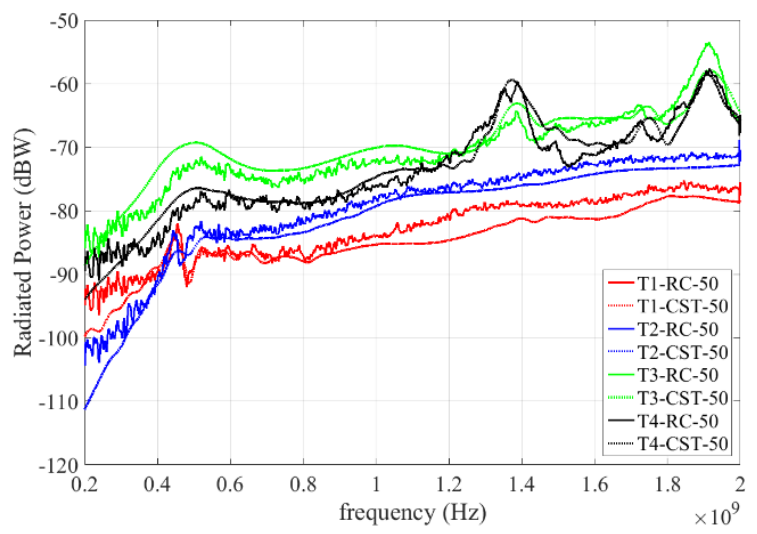

Fig. 4. RP with a $50 \Omega$ load in measurement (Tx-RC-50) and simulation (Tx-CST-50), $\mathrm{x}=1,2,3$ or 4 


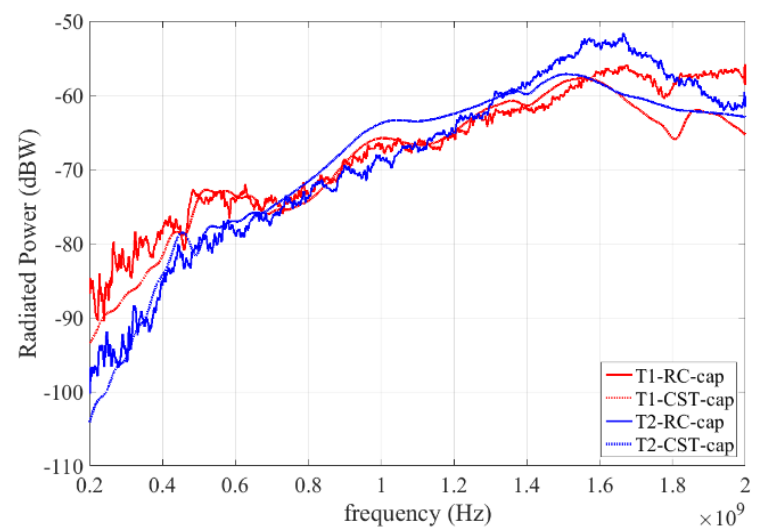

Fig. 5. RP with a floating capacitive load in measurement (Tx-RC-cap) and simulation (Tx-CST-cap), $\mathrm{x}=1,2$

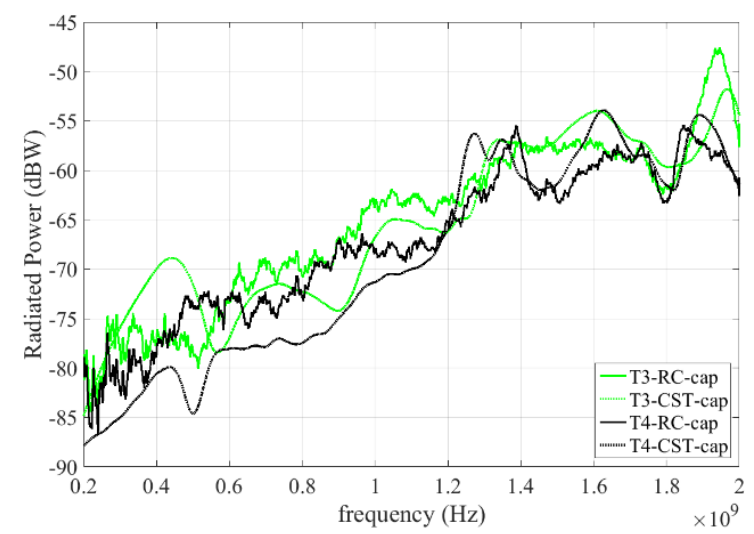

Fig. 6. RP with a floating capacitive load in measurement (Tx-RC-cap) and simulation (Tx-CST-cap), $\mathrm{x}=3,4$

Fig. 4 exhibits satisfying results as levels and variations are similar in simulation and measurement for all scenarii. Looking more carefully at Fig. 4, T1 and T2 have the same trend while $\mathrm{T} 3$ and $\mathrm{T} 4$ have a different one, with resonances near $1.4 \mathrm{GHz}$ and $1.9 \mathrm{GHz}$. These resonances come from plane resonances. Indeed, T3 and T4 are routed above 2 successive ground planes linked by only some vias far from the tracks. On the contrary, T1 and T2 are routed over a single ground plane, a configuration which avoids plane resonances. These results show a good ability of the solver to represent the reality. However, to perform a fair parametric study, the same configuration of stack-up for all scenarii will be kept in Section IV. The stack-up of T1 and T2 will be selected to get rid of ground plane resonances in this study.

We can see on Fig. 4 that the scenarii are quite well sorted for all frequencies. The most radiating scenario is T3, a matched line with high $h$. The fact this line radiates more than the other one with a smaller line width (T4, $94 \Omega)$ is surprising. However, both numerical and experimental results confirm this. This was verified numerically on some cases with a program based on Green's function and transmission line theory[3]. For all scenarii, the power is mainly radiated by the vertical current (in the vias) because track and ground plane are close to each other. Determining how line width influences the radiation of the currents in vias would demonstrate this phenomenon, but it is not so easy to tackle this problem analytically. Scenario T3 radiates more than $\mathrm{T} 1$ because it is 3 times farther from the ground plane. Difference of RP expected is $9.5 \mathrm{~dB}$ for all frequencies, for either simulation or measurement results, if we ignore ground plane resonances. Scenario T1 radiates more than T2 below $450 \mathrm{MHz}$. Low frequency result is intended [4] as RP of a current loop is proportional to the square of the area of the loop. However, at $200 \mathrm{MHz}$, the RP difference between $\mathrm{T} 1$ and $\mathrm{T} 2$ reaches only $10 \mathrm{~dB}$ (and not $20 \mathrm{~dB}$ ). It may be due to the contribution to the current path in the SMA connectors. In high frequency, results are more startling, but were also confirmed by the radiation model based on transmission line theory and Green's function formalism for estimation of radiated emission[3]. It confirms that for electrically long wires the radiation pattern exhibit more side lobes as the frequency increases, and that the RP follows the fluctuations of the equivalent radiation resistance.

Fig. 5 and Fig. 6 represent the same comparison with another load. Due to manufacturing mistake, the capacitance at the load is floating, so the simulation was made in the same way. Simulations and measurements exhibit some common features but the comparison is not satisfying enough. We suspect that the electrical model of the load is responsible for that discrepancy, given the fact that the only change in the measurement and simulation was the substitution of matched impedance for a capacitor (mounted on small PCB for measurements). Floating load probably add difficulties. This will be further investigated by new measurements with a connected capacitance. Meanwhile, a perfect capacitor model is retained for the following analysis of its sensitivity with regard to RP.

\section{SURROGATE MODEL}

Surrogate models describe the behavior of outputs as a function of input variables. Their construction is based on a limited set of realizations of the initial model. Many types of surrogate functions exist. Kriging was selected in this study[5] among other possible methods. Kriging describes the result as a random process as in (2).

$$
R P=\mu+\varepsilon(L, w, h, C, f)
$$

where $\mu$ is the mean, which is supposed to be constant (ordinary kriging). $\varepsilon$ is a gaussian process of input variables (supposedly random variables) which is find by optimization on variance from known values. The only condition for applying such a method is that RP may be described by a stationary process with regard to its domain of variation. We used it as a trial and error procedure. Failure would mean to look for another surrogate model such as polynomial chaos for instance.

\section{A. Methodology of extraction}

Due to simulation time and tool capacity, the design of experiment (DoE) is limited to 200 different simulations, generated with the Latin Hypercube Sampling (LHS) for the independently and uniformly distributed $\mathrm{L}, \mathrm{w}, \mathrm{h}$ and $\mathrm{C}$ parameters. The frequency was added with a linear sampling (51 values) since, thanks to the time domain solver, more frequency points can be added without increasing simulation time. Then the DoE is simulated on CST MICROWAVE STUDIO ${ }^{\circledR}$, to estimate the 10200 values of RP. This values are imported in Matlab ${ }^{\circledR}$ to feed the surrogate model, implemented with the uqlab tool[6]. The algorithm used to build the surrogate model cannot process a too big dataset, which is why the linear sampling in frequency was limited to 51 points. RP input values were scaled in $\mathrm{dBW}$ rather than 
on linear scale. It enhanced the performance of the kriging model.

\section{B. Self-consistency of surrogate model}

With kriging, the response on the DoE used for the learning fits exactly. So another DoE is generated, with 20 cases in LHS for the four first parameters and a linear sampling for the frequency (901 points between $200 \mathrm{MHz}$ and $2 \mathrm{GHz}$ ). CST simulates this DoE whereas RP is calculated from the surrogate model.

Fig. 7 shows some comparison of RP levels as a function of frequency between CST simulation and surrogate model prediction. The surrogate model gives good trends and levels in each case. To quantify the surrogate model performance, the distribution of the relative error (applied to linear values of RP) is presented in Fig. 8. Its standard deviation is $25 \%$, which correspond to a difference of $1 \mathrm{~dB}$. This is reasonable regarding to the dynamic range of the different scenarii.

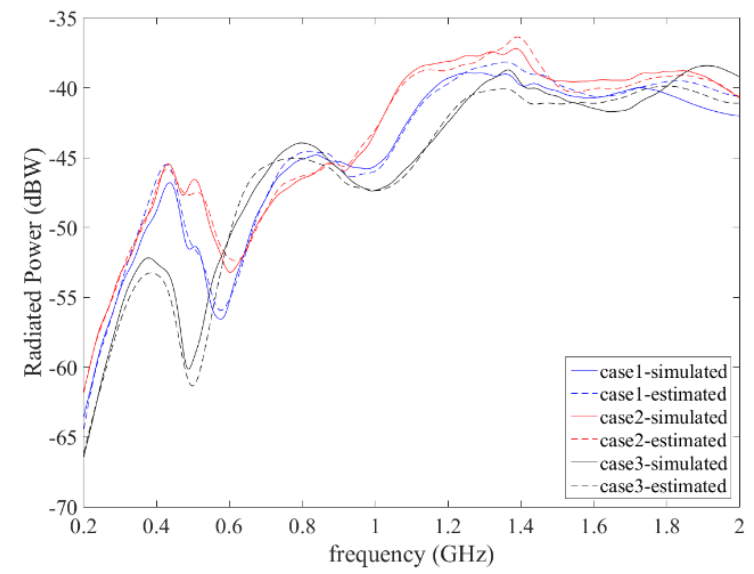

Fig. 7. Simulation vs surrogate model for 3 cases on 20 tested

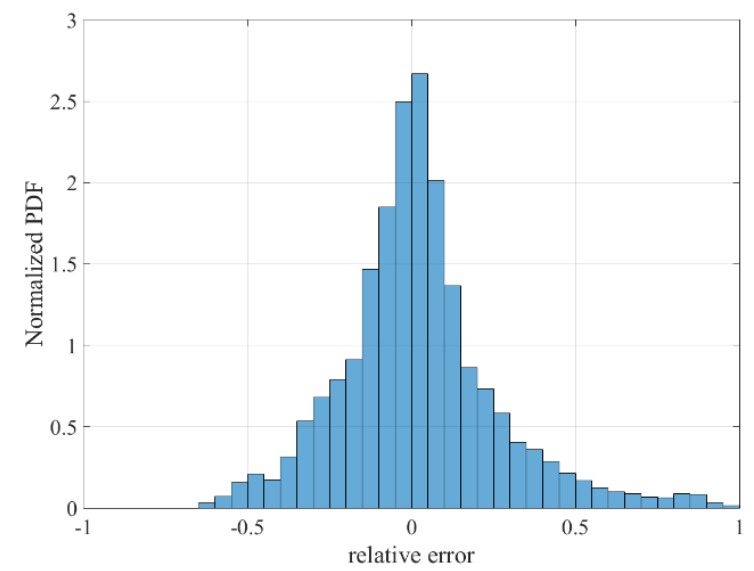

Fig. 8. Distribution of the relative (linear) error between model and simulation

\section{SENSITIVITY ANALYSIS}

From the surrogate model the Sobol' indices can be extracted to see which parameters contribute the most to the variations of RP. To do so a LHS sample of 10000 realizations for the fifth variables is generated and evaluated by means of the surrogate model. As shown in Fig. 9 and Fig. 11, the frequency is clearly the most important parameter, even if this result can be slightly biased because of the sampling of the frequency, finer than the other parameters sampling, in the data used for the learning. The dielectric height also has an influence at the first order, as the couple (L, f) at the second order (Fig. 10). These two last parameters mutually explain partially the RP in addition to the contributions of $h$ and above all, $f$. Only 5\% of the variance is explained at superior order combinations of input variables.

To supplement these results, another DoE of 10000 realizations is created. Only the $500(5 \%)$ highest values of $\mathrm{RP}$ are retained, so that the sensitivity of extreme values of $\mathrm{RP}$ is investigated. The fifth boxplot in Fig. 12 confirms the influence of the frequency, which is more concentrated at the highest values of frequency. Therefore, the higher the frequency the more the track radiates. Other variables less matter. The high values of the median and quartiles of $h$ also confirm its first order influence. Therfore, when defining a stack-up, taking $h$ as low as possible is the priority, while keeping impedance constant if needed for signal integrity. Even if Sobol' indices indicates that $w$ has the minimum influence, its boxplot gives more importance to high value. In case of higher $h$ values (scenarii T3 and T4), lowering $w$ reduces the RP at most frequencies. That may explain that T4 (with low $w$ and large characteristic impedance) does not radiate much than $\mathrm{T} 3$ but at some frequency ranges only.

Fig. 13 gives some insight about the role of $h / w$, which decreases and increases with line characteristic impedance. It confirms that low $\mathrm{h} / \mathrm{w}$ ratio $(<0.5)$ values gives less high radiating scenarii compared to the whole sample. However, it also indicates that very high $\mathrm{h} / \mathrm{w}$ ratio $(>2.5)$ scenarii provide less, or at least not more extreme RP values than the general sample.

\section{CONCLUSION}

In this paper the ability of kriging to modelize the radiated power of a microstrip line has been demonstrated. Some information has been extracted from the surrogate model in terms of sensitivity, which can guide PCB conception. The frequency is by far the main parameter, so signal properties is the first parameter an EMC engineer will focus on. Then the height of dielectric is the most important design parameter, to decrease as much as possible. Even if this parameter is not the most influent, it is interesting to note we may decrease line width to densify a board and reduce or at least do not increase RP, but in the limit of signal integrity requirements.

Beyond the sensitivity analysis, the model can also be used to quickly compare two scenarii during stack-up conception. It is then possible to evaluate a risk/reward ratio from more quantitative data, which are impossible to get from a global parametric study.

Some improvements can be performed. The correlation between simulations and measurements for capacitive load has to be improved in particular. The model itself could be enhanced to match more to the reality: RLC model of the load could be used without all the connectors. A lower impedance at the source (when no adaptation is performed on the line) is also an interesting case study, which will be more resonating.

This paper highlights, based on a simple scenario, that the methodology presented is relevant to find most sensitive parameters in a design. This methodology will be further extended to study a set of pre-identified critical scenarii. 


\section{ACKNOWLEDGMENT}

This project was also supported in part by the European Union through the European Regional Development Fund, in part by the Ministry of Higher Education and Research, in part by the Région Bretagne, and in part by the Département d'Ille et Vilaine and Rennes Métropole, through the CPER Project SOPHIE/STIC \& Ondes.

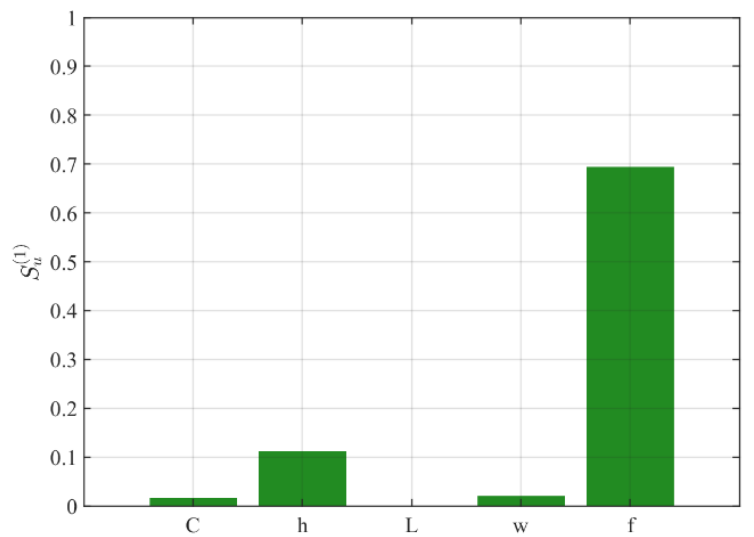

Fig. 9. Order 1 of the Sobol Analysis

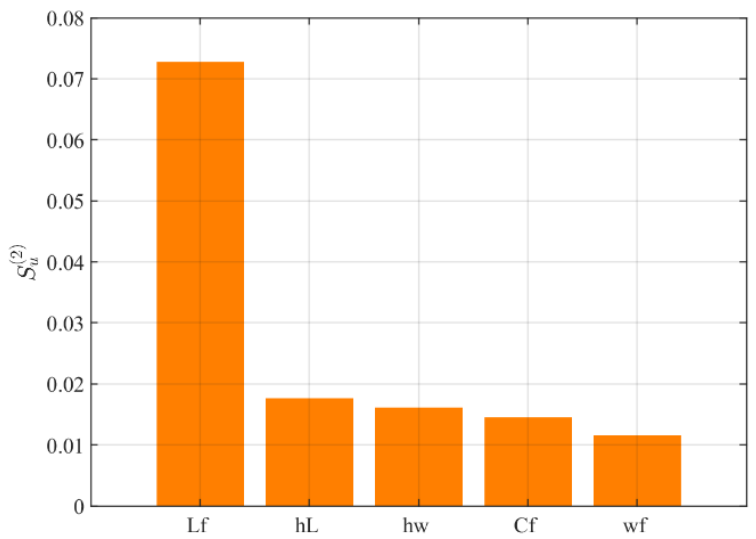

Fig. 10. Order 2 of the Sobol Analysis

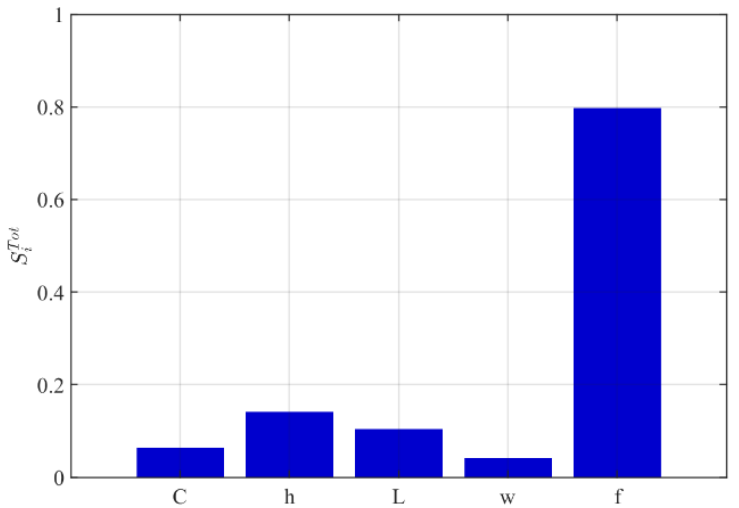

Fig. 11. Total influence of each variable in Sobol Analysis

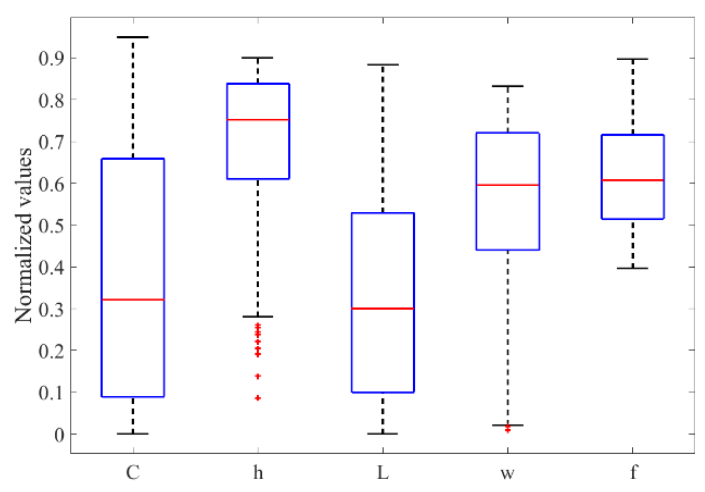

Fig. 12. Distribution of 500 most radiating scenarii out of 10000

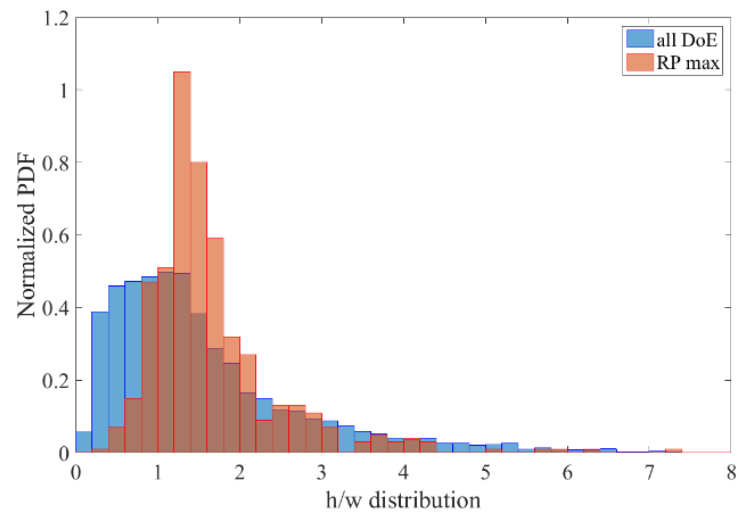

Fig. 13. h/w distribution of all DoE and 5\% most radiating samples

\section{REFERENCES}

[1] J. M. Hock et J. D. Prymak, «Inductance of Bypass Capacitors: How to Define, How to Measure, How to Simulate », p. 46.

[2] C. L. Holloway, H. A. Shah, R. J. Pirkl, W. F. Young, D. A. Hill, et J. Ladbury, "Reverberation Chamber Techniques for Determining the Radiation and Total Efficiency of Antennas », IEEE Trans. Antennas Propag., vol. 60, n 4, p. 1758-1770, avr. 2012.

[3] M. Leone, «Closed-Form Expressions for the Electromagnetic Radiation of Microstrip Signal Traces », IEEE Trans. Electromagn. Compat., vol. 49, $\mathrm{n}^{\mathrm{o}} 2$, p. 322-328, mai 2007.

[4] M. Mardiguian, Controlling radiated emissions by design. New York: Springer Science+Business Media, LLC, 2001.

[5] M. A. Oliver et R. Webster, «A tutorial guide to geostatistics: Computing and modelling variograms and kriging », CATENA, vol. 113, p. 56-69, févr. 2014.

[6] R. Schöbi, S. Marelli, et B. Sudret, «UQLab User Manual- PC-Kriging », p. 27. 